\title{
Estimation de la part du ruissellement sur les versants dans les crues du ruisseau du Rimbaud (massif des Maures, Var, France) après l'incendie de forêt d'août 1990
}

\author{
C. MARTIN \\ Centre Aixois de Géographie Physique, CAGÉP-URA 903 du CNRS, Institut de Géographie \\ de l'Université de Provence, F-13621 Aix-en-Provence cedex 1, France.
}

\section{J. LAVABRE}

Cemagref, Le Tholonet, BP 31, F-13612 Aix-en-Provence cedex 1, France.

\begin{abstract}
Résumé : Les recherches sur l'hydrologie, l'hydrochimie et les phénomènes d'érosion mécanique menées dans le bassin versant du Rimbaud $\left(1,46 \mathrm{~km}^{2}\right)$ après l'incendie de forêt d'août 1990, ont permis la mise au point d'une méthode de décomposition des hydrogrammes entre les eaux fournies par le ruissellement sur les versants et les eaux ayant effectué un trajet souterrain. La décomposition utilise comme traceur le sodium ou les ions chlorures, deux éléments dont les concentrations mesurées dans la pluie ne subissent pas de modification au contact du sol après la destruction du couvert végétal. Les essais conduits avec l'oxygène-18 n'ont pas été concluants. Deux crues, les plus représentatives du comportement impulsionnel du ruisseau du Rimbaud après l'incendie, ont été décomposées. L'une s'est produite en début d'année hydrologique (27 septembre $1992-\mathrm{P}: 72,0 \mathrm{~mm}$ ), l'autre à la fin des pluies d'automne (9 décembre $1990-\mathrm{P}: 87,7 \mathrm{~mm}$ ). Ces deux épisodes diffèrent très nettement par l'abondance des écoulements générés par les précipitations (coefficient d'écoulement total : 18,6\% en septembre $1992 ; 87,5 \%$ en décembre 1990). Mais elles se ressemblent par les débits maxima $\left(7,7\right.$ et $\left.7,8 \mathrm{~m}^{3} \cdot \mathrm{s}^{-1}\right)$ et par l'importance des eaux fournies par le ruissellement sur les versants pendant la crue elle-même. Cette source d'alimentation représente $89 \%$ du débit de pointe et $76 \%$ du volume d'eau écoulé pendant la crue du 27 septembre $1992 ; 89 \%$ du débit de pointe et $47 \%$ du volume d'eau écoulé pendant la crue du 9 décembre 1990.
\end{abstract}

\section{An estimation of the contribution of slope runoff to the Rimbaud stream floods (massif des Maures, Var, France) after the August 1990 forest fire}

Abstract: Following the August 1990 forest fire on the Rimbaud catchment $\left(1.46 \mathrm{~km}^{2}\right)$, studies on hydrological, hydrochemical and erosion response led to the development of a decomposition method in hydrograph separation between the waters originating from slope runoff and those originating from underground flow. Sodium and chloride ions were used as tracers, since they are two elements whose concentrations measured in rainfall do not undergo any change on contact with the ground after the vegetation cover has been destroyed. Tests with ${ }^{18} \mathrm{O}$ were inconclusive. Two of the floods that most typified the post-fire surging characteristics of the Rimbaud stream were chosen for decomposition, one at the beginning of the hydrological year (27 September 1992 with a rainfall of $72.0 \mathrm{~mm}$ ) and the other towards the end of the autumn rains ( 9 December 1990 with a rainfall of $87.7 \mathrm{~mm}$ ). These two flood events show clear differences in the volume of runoff originating from rainfall (total runoff coefficient: $18.6 \%$ for September 1992 and $87.5 \%$ for December 1990), but similarities in .maximum discharge $\left(7.7\right.$ and $\left.7.8 \mathrm{~m}^{3} . \mathrm{s}^{-1}\right)$ and in the quantities of water yielded by slope runoff during the flood itself. The latter source represented $89 \%$ of the peak flow and $76 \%$ of the total flow during the September 1992 flood, and $89 \%$ of the peak flow and $47 \%$ of the total flow during the December 1990 flood. 


\section{INTRODUCTION}

En août 1990, un incendie a détruit 8400 ha de forêt et de maquis dans la partie occidentale du massif des Maures, affectant partiellement le Bassin Versant de Recherche et Expérimental (BVRE) du Réal Collobrier (Fig. 1), géré par le Cemagref depuis 1966. Les recherches immédiatement engagées ont permis de cerner les conséquences du feu sur l'hydrologie (Lavabre et al., 1993), l'hydrochimie (Martin, 1995) et les phénomènes d'érosion mécanique (Martin et al., 1995)., en relation avec un suivi de la reprise végétale (Viné \& Puech, 1994).

Les recherches les plus fines ont été conduites sur le bassin versant du Rimbaud (Fig. 1). La décomposition des hydrogrammes y a été abordée selon deux orientations :

- D'une part, pour distinguer les eaux "nouvelles" des eaux "anciennes" (Travi et al., 1994), en utilisant un traceur chimique (ions chlorures) ou isotopique (oxygène-18). Cette approche est maintenant classique en hydrologie (Blavoux et al., 1976; Hooper \& Schoemaker, 1986).

- D'autre part, pour différencier les eaux directement fournies aux ruisseaux par le ruissellement sur les versants des eaux ayant effectué un trajet souterrain. C'est ce sujet qui est traité dans le présent article.

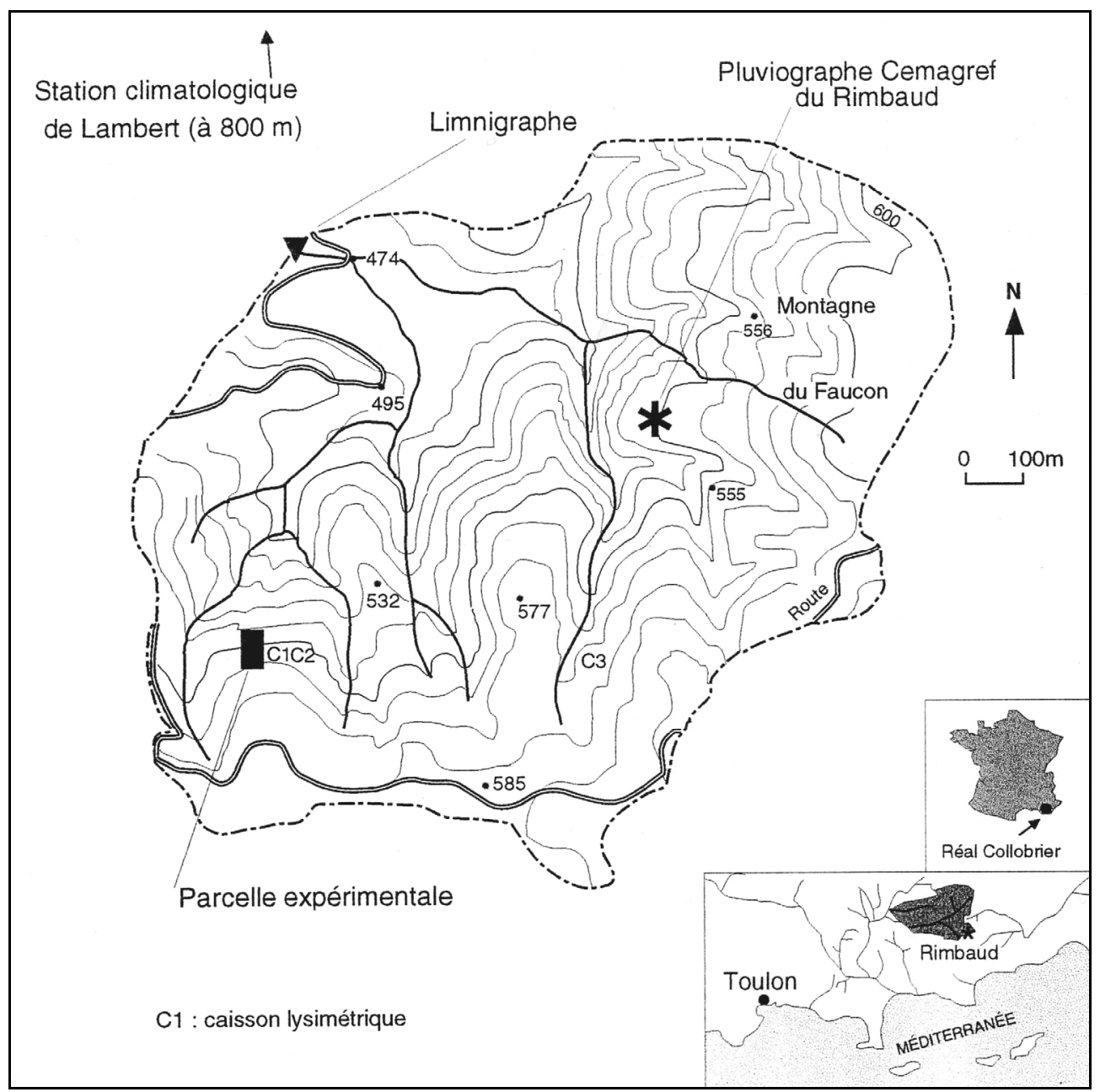

Fig. 1 Localisation et présentation du bassin versant du Rimbaud. 


\section{LE TERRAIN D'ÉTUDE}

Le bassin du Rimbaud $\left(1,46 \mathrm{~km}^{2}\right)$ est constitué de gneiss massifs affectés d'un très fort pendage vers le nord-ouest. Les altitudes sont comprises entre 470 et $622 \mathrm{~m}$ Les versants présentent une pente moyenne de l'ordre de $9-10^{\circ}$. Les pentes sont très faibles sur les croupes sommitales et à l'extrémité aval du bassin versant. Mais leur inclinaison dépasse souvent $20^{\circ}$ dans les gorges creusées dans le cours moyen des ruisseaux. Dans ces secteurs, les gneiss affleurent très fréquemment. Les sols - peu épais, caillouteux, à texture sableuse, et à structure grumeleuse à submotteuse - appartiennent à la classe des rankers. Ils recouvrent généralement des roches altérées cohérentes. Avec des précipitations annuelles moyennes supérieures à $1100 \mathrm{~mm}$ et une température moyenne de $12{ }^{\circ} \mathrm{C}$ environ, le bassin versant connaît un climat de type méditerranéen humide. La massiveté des roches, la minceur des formations superficielles et les caractères morphométriques du bassin versant $(K c$ de Gravelius $=1,07 ; D d=4,86 \mathrm{~km} \cdot \mathrm{km}-2 ; R c$ de Strahler $=2,81)$ s'associent pour que le ruisseau du Rimbaud réagisse violemment aux précipitations. En revanche, l'écoulement s'interrompt pendant une partie de l'été. Sur la période août 1967 - septembre 1990, la lame d'eau écoulée annuelle moyenne s'élève à $634 \mathrm{~mm}$.

L'incendie d'août 1990 a parcouru 84 \% du bassin versant (Puech et al., 1991), en détruisant un maquis complanté de chênes lièges et de pins maritimes. Une petite châtaigneraie, située dans la partie aval du bassin versant, a échappé au feu. L'équipement qui était en place au moment de l'incendie (limnigraphe, pluviographe, préleveur automatique d'échantillons) a permis d'être immédiatement opérationnel. La station de mesure des écoulements est équipée d'un déversoir triangulaire qui assure une bonne précision même pour les forts débits. L'enregistrement des hauteurs d'eau et des précipitations est effectué sur support électronique depuis 1988. Pendant les crues, le rythme des prélèvements automatiques était de un toutes les 30 minutes. Des prélèvements manuels ont en outre été effectués lors des passages sur le terrain.

Le dispositif expérimental a en outre été complété par une parcelle de mesure de l'érosion des sols (Fig. 1). La parcelle $\left(75,2 \mathrm{~m}^{2}\right.$ pour une longueur de $12 \mathrm{~m}$ ) a la forme d'un parallélogramme délimité par une double rangée de tôles enfoncées d'une quinzaine de centimètres. La pente avoisine $11^{\circ}$. La parcelle est reliée par une gouttière à une batterie de deux cuves réceptrices dont la première sert de partiteur au 1/9. Le dispositif est complété par un pluviographe. Le sol a une épaisseur de $20 \mathrm{~cm}$ environ. Le couvert végétal (bruyères arborescentes, arbousiers, jeunes pins maritimes) a été entièrement détruit par le feu. La revégétalisation, essentiellement à base de pins, s'est révélée beaucoup plus lente que sur l'ensemble du bassin versant.

Au cours de l'automne 1991, une dizaine d'hectares situés dans la partie inférieure du bassin versant ont été raclés au bulldozer puis sous-solés par l'Office National des Forêts, afin de préparer une plantation. Par ailleurs, en janvier et février 1991, les arbres calcinés et les repousses des essences du maquis ont été éliminés (à l'aide de tracteurs gyrobroyeurs ou par débitage manuel) sur près de 70 ha.

\section{LES OBSERVATIONS HYDROLOGIQUES}

La destruction du couvert végétal s'est traduite, au cours des trois premières années après l'incendie, par une augmentation de la lame d'eau écoulée annuelle. Ce supplément, qui 
représente autour de 12-15\% des précipitations (Lavabre et al., 1996), s'est surtout manifesté lors des crues :

- En 1990-91, le comportement du ruisseau du Rimbaud est devenu fortement impulsionnel. Les débits de pointe ont dépassé à trois reprises le débit décennal calculé pour la période avant incendie $\left(5 \mathrm{~m}^{3} \cdot \mathrm{s}^{-1}\right)$; et le débit maximal a atteint $7,8 \mathrm{~m}^{3} \cdot \mathrm{s}^{-1}$, le 9 décembre, pour des précipitations ne présentant aucun caractère exceptionnel, ni par leur abondance $(88,0 \mathrm{~mm})$ ni par leur intensité (intensité maximale en 6 minutes de l'ordre de $40 \mathrm{~mm} \cdot \mathrm{h}^{-1}$ ). Les montées de crue ont souvent demandé moins de cinq minutes, et les décrues ont été elles-mêmes très rapides. Le caractère excessif pris par les réactions du cours d'eau aux précipitations s'explique par le ruissellement sur les versants mis à nu par l'incendie. L'année 1991-92 n'a connu qu'un seul épisode violent $\left(3,6 \mathrm{~m}^{3} . \mathrm{s}^{-1}\right.$, en octobre), mais plusieurs crues spectaculaires se sont encore produites en 1992-93 $\left(7,7 \mathrm{~m}^{3} \cdot \mathrm{s}^{-1}\right.$ en septembre; $3,5 \mathrm{~m}^{3} \cdot \mathrm{s}^{-1}$ en octobre ; $4,3 \mathrm{~m}^{3} . \mathrm{s}^{-1}$ en novembre). En 1993-94, le débit maximal a atteint $2,2 \mathrm{~m}^{3} \cdot \mathrm{s}^{-1}$ seulement. La reprise végétale est alors intervenue pour amortir la réponse des écoulements aux précipitations (taux de recouvrement des surfaces incendiées par les arbustes : $15 \%$ en août 1991, 30-35\% en août 1992 et 45-50 \% en août 1993 - d'après les observations de Puech et al. (1994) et Viné (communication personnelle).

- Les débits de pointe les plus élevés de la période post-incendie ont été générés par des pluies d'un volume très modéré pour le terrain d'étude (Fig. 2).

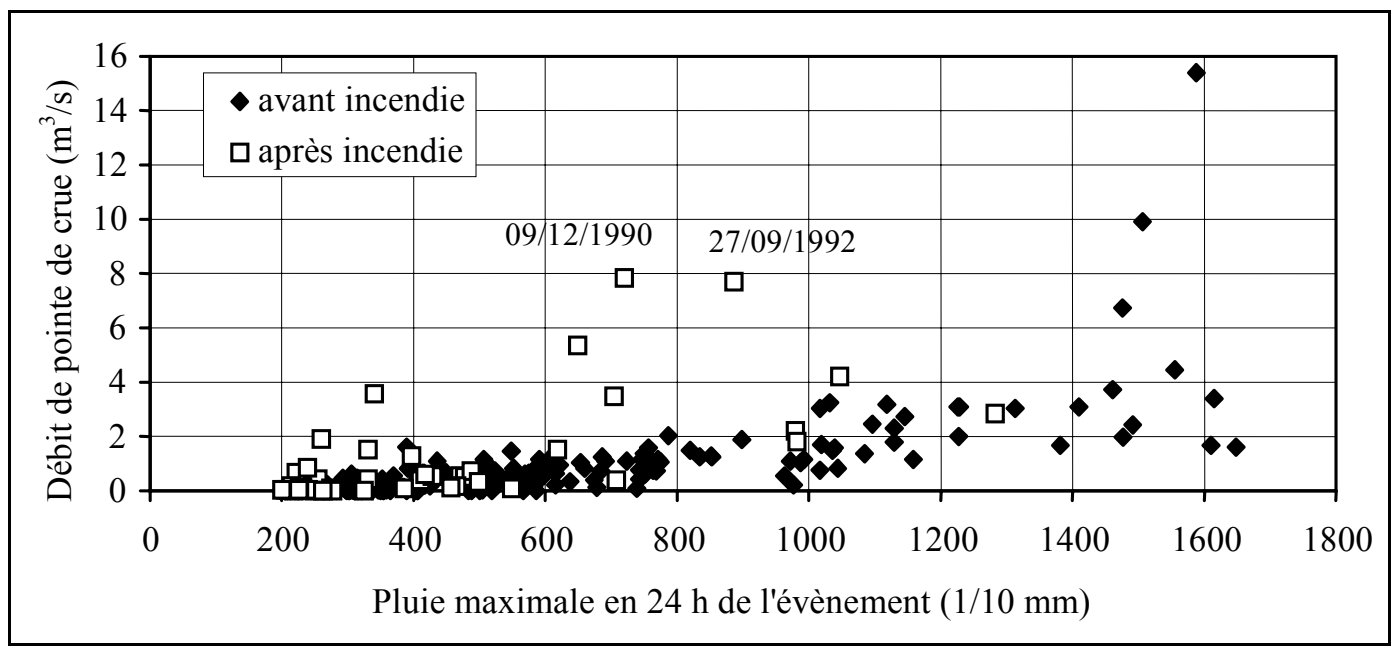

Fig. 2 Relations en automne (septembre, octobre et novembre) entre les débits de pointe de crue et les précipitations en 24 heures (période après incendie : septembre 1990 - novembre 1994).

- La comparaison des débits moyens journaliers observés du 26 octobre 1990 au 31 décembre 1991, avec les débits simulés sur la même période en utilisant le modèle de prévision des écoulements GR3 (Michel et al., 1991), bien calé sur les données recueillies avant l'incendie (Lavabre et al., 1993), fait ressortir les effets de la disparition du couvert végétal en périodes pluvieuses (Fig. 3). 


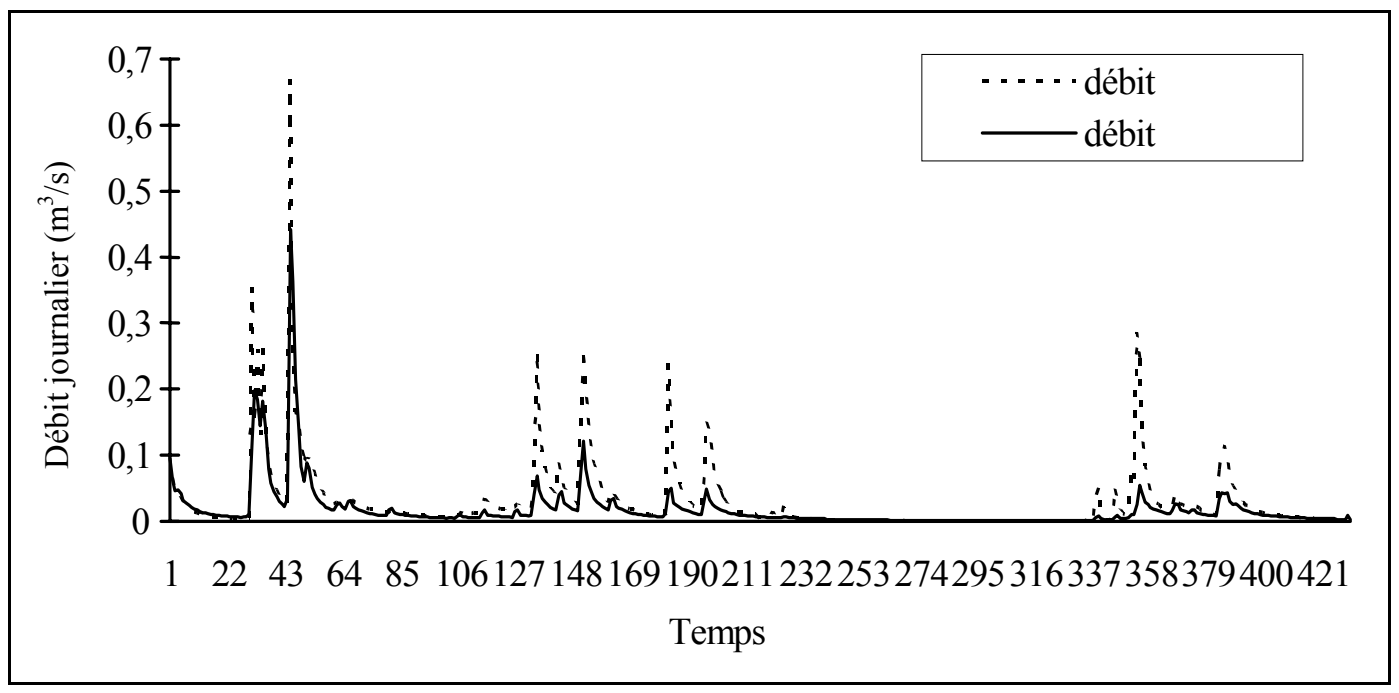

Fig. 3 Comparaison des débits journaliers mesurés après l'incendie avec les valeurs reconstituées à partir des informations recueillies avant le feu (la période représentée démarre le 26 octobre 1990).

\section{LA MÉTHODE DE DÉCOMPOSITION DES HYDROGRAMMES}

La décomposition doit s'appuyer sur un traceur, chimique ou isotopique, dont la concentration diffère sensiblement entre les eaux de ruissellement superficiel et les eaux ayant effectué un trajet souterrain. Par ailleurs, pour l'ensemble d'un bassin versant, il est difficile de connaître la composition chimique des eaux de ruissellement. On est généralement obligé de l'identifier à celle des précipitations. La concentration du traceur ne doit donc pas subir de modification à la surface du sol. Enfin, le résultat de la décomposition sera d'autant plus satisfaisant que la concentration du traceur dans la pluie demeurera stable pendant l'épisode étudié.

Deux traceurs ont été retenus : le sodium et les ions chlorures. En terrain dénudé, les concentrations de ces deux éléments n'augmentent pas à la surface du sol (voir infra). Après la destruction du couvert végétal, qui constitue un piège à aérosols particulièrement efficace, les pluviographes à ouverture horizontale fournissent des indications fiables sur les entrées réelles de ces éléments dans les bassins versants. Le sodium a été dosé par photométrie de flamme et les ions chlorures par volumétrie (en 1990-91 - dosage par le nitrate de mercure) puis, après vérification de la bonne correspondance avec les analyses volumétriques, par colorimétrie (avec l'auto-analyseur Technicon). L'oxygène-18 peut également être utilisé (détermination par spectométrie de masse après équilibration avec $\mathrm{CO}_{2}$ ), mais il faut que le marquage de la pluie soit très différent de celui des eaux d'écoulement souterrain, ce qui n'est pas toujours le cas.

La première étape de la démarche consiste à déterminer, selon une approche hydrologique classique, le débit du ruisseau pour lequel les écoulements ne sont plus influencés par le ruissellement sur les versants. Dans le bassin versant du Rimbaud, le choix du point d'inflexion sur l'hydrogramme de décrue représenté en coordonnées semilogarithmiques, a pu être parfois conforté par les mesures des transports solides.

Les prélèvements effectués permettent d'estimer la composition chimique des eaux du ruisseau pour n'importe quel point de l'hydrogramme, en utilisant les régressions 
(généralement bi-logarithmiques) liant les concentrations et les débits dans la plupart des bassins versants de roches cristallines. Dans un premier temps (Martin et al., 1994), nous avons effectué la décomposition à partir de la concentration du traceur dans l'eau de pluie, de sa concentration dans l'eau du ruisseau pour tous les points de l'hydrogramme ayant servi à calculer les volumes écoulés, et de sa concentration au point d'inflexion marquant la fin du ruissellement sur les versants. Pour chaque point de l'hydrogramme, on pose (équation 1) :

$$
Q i S=Q i T(C T-C P) /(C S-C P)
$$

où $Q i S$ est le débit instantané des eaux ayant effectué un trajet souterrain, QiT le débit instantané du ruisseau, $C T$ la concentration instantanée du traceur dans les eaux du ruisseau, $C P$ la concentration du traceur dans la pluie et $C S$ la concentration du traceur au point d'inflexion marquant la fin du ruissellement sur les versants.

L'approche a subi, depuis, une modification. En effet, nous étions partis de l'idée, acquise avant incendie, que chaque phase de récession devait fournir, pour les valeurs de débit qui nous intéressent, une seule série de régressions entre la composition chimique des eaux et l'écoulement instantané (Martin, 1987). Or il est apparu qu'après incendie, deux séries pouvaient être établies, et que le passage de l'une à l'autre se produisait vraisemblablement lorsque le débit du ruisseau n'était plus influencé par le ruissellement sur les versants. Dans ces conditions, QiS est déterminé de la manière suivante :

1. Pour chaque décrue, il est possible d'intégrer à l'équation (1) la régression liant les concentrations $C S$ au débit $Q i S\left[\log C S=\mathrm{a} \log Q i S+\mathrm{b}\right.$; soit $C S=10^{\mathrm{b}}$. QiS $S^{a}$. On aboutit ainsi à une équation dont la résolution permet de déterminer $Q i S$ :

$\left.Q i S\left[10^{b} \cdot Q i S^{a}\right)-C P\right]=Q T(C T-C P)$

L'opération étant assez lourde, nous lui avons préféré une démarche itérative. Une fois calculées la concentration $C S$ et la concentration $C T$ correspondant au point de l'hydrogramme considéré, l'équation (1) (voir supra) fournit une première valeur de QiS. Il est alors possible de déterminer la concentration $C S^{\prime}$ (concentration du traceur dans les eaux fournies par l'écoulement souterrain) spécifique du point de l'hydrogramme considéré, en utilisant la régression concentrations-débits définie après la fin du ruissellement sur les versants. En remplaçant $C S$ par $C S^{\prime}$ dans l'équation (1), on détermine une valeur améliorée de QiS. L'opération est renouvelée jusqu'à ce que le débit "profond" calculé soit stabilisé.

2. Pour chaque montée de crue, on dispose de la concentration du traceur (souvent extrapolée) au début de la montée des eaux. On connaît également les concentrations en pointe de crue $C T$ (généralement extrapolée à partir de la décrue) et $C S^{\prime}$ (extrapolée à partir de la deuxième partie de la récession). Même en l'absence de prélèvement pendant la montée de crue, il est donc possible d'établir les régressions concentrations-débits nécessaires à la décomposition. Le calcul est ensuite mené comme pour les décrues. Lorsque des analyses en montée de crue sont disponibles, les régressions se révèlent généralement de moins bonne qualité qu'en décrue. Les concentrations $C T$ sont alors estimées en traitant séparément chaque partie de l'hydrogramme comprise entre deux points de prélèvement. Les volumes d'eau écoulés en montée de crue sont heureusement beaucoup plus faibles qu'en décrue.

3. Les débits $Q i S$ trouvés pour tous les points de l'hydrogramme utilisés pour déterminer les écoulements du ruisseau, servent à déterminer les volumes d'eau ayant effectué un trajet souterrain. Les volumes d'eau fournis par le ruissellement sur les versants sont obtenus par différence. 


\section{LA DÉCOMPOSITION DES DEUX PRINCIPALES CRUES}

Les observations sur l'érosion chimique et mécanique, menées par le Centre Aixois de Géographie Physique, dans le bassin versant du Rimbaud, n'étaient pas orientées vers la résolution de problèmes hydrologiques. C'est du reste l'étude de l'érosion hydrique et des modalités de fourniture des matériaux aux cours d'eau qui nous a posé le problème de l'ampleur du ruissellement sur les versants. Le suivi n'était donc pas adapté aux nécessités d'une approche dont l'élaboration s'est faite a posteriori. Les crues du 9 décembre 1990 et du 27 septembre 1992 ont cependant fourni des données suffisantes pour réaliser la décomposition. Celle-ci est d'autant plus facile, et d'autant plus instructive, que, comme ce fut le cas pendant toute la période d'observation, les roches altérées et les sols n'ont pas été portés à saturation: le retour en surface d'eaux infiltrées n'est donc pas venu se surimposer au ruissellement directement déclenché par les eaux pluviales. Les quantités d'eau soustraites à l'infiltration par le ruissellement sur les versants ont favorisé ce type de fonctionnement.

\section{La crue du 9 décembre 1990}

Cette crue présente deux pics (Fig. 4) en relation avec des pluies d'intensité moyenne. Le premier pic $\left(1,8 \mathrm{~m}^{3} \cdot \mathrm{s}^{-1}\right)$ fait suite à une averse de $13,2 \mathrm{~mm}$ en 30 minutes; et le second $\left(7,8 \mathrm{~m}^{3} \cdot \mathrm{s}^{-1}\right)$ à une averse de $31,2 \mathrm{~mm}$ en $1 \mathrm{~h} 10$. Les temps de réponse avoisinent 20 minutes. Les précipitations des 8 et 9 décembre $(87,7 \mathrm{~mm})$ ont été précédées par des pluies d'automne moyennement abondantes $(41 \mathrm{~mm}$ en septembre, $231 \mathrm{~mm}$ en octobre et $133 \mathrm{~mm}$ en novembre). Le débit maximal a dû être reconstitué à partir de l'observation des laisses de crue, la montée du flotteur du limnigraphe ayant été bloquée. L'horaire attribué à la pointe de crue souffre également d'une certaine incertitude.

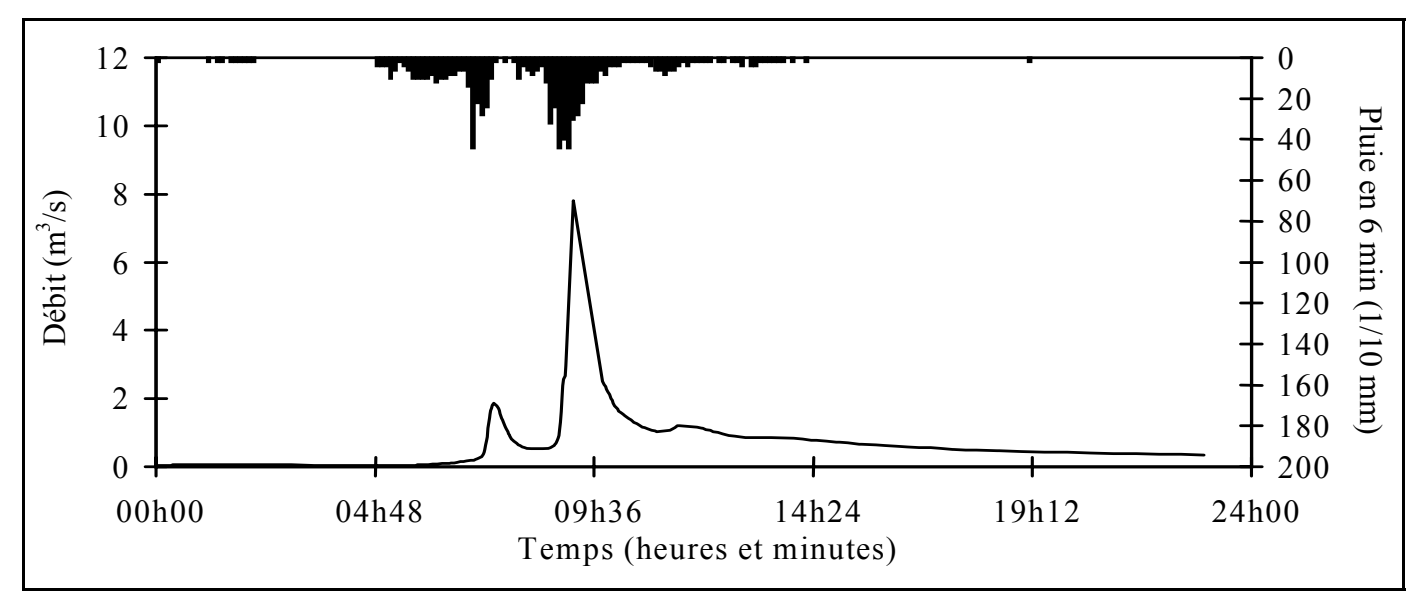

Fig. 4 La crue du 9 décembre 1990.

La décomposition s'appuie sur les ions chlorures qui ont fait l'objet du suivi le plus serré (18 dosages sur les eaux prélevées les 8 et 9 décembre, dont un pour un débit de $4,4 \mathrm{~m}^{3} / \mathrm{s}$ - Tableau 1). Les concentrations mesurées dans les eaux de pluie (prélevées le 12) témoignent d'une assez bonne homogénéité spatiale $\left(2,1 \mathrm{mg} . \mathrm{l}^{-1}\right.$ au pluviographe Cemagref du Rimbaud ; $1,9 \mathrm{mg} . \mathrm{l}^{-1}$ à la station de Lambert). L'homogénéité dans le temps est en outre attestée par la concentration de $1,8 \mathrm{mg} . \mathrm{l}^{-1}$ mesurée dans les eaux de ruissellement de la parcelle expérimentale (coefficient de ruissellement : $12 \%$ - du 8 au 12 décembre). La valeur de $1,9 \mathrm{mg} . \mathrm{l}^{-1}$ a été retenue pour caractériser les eaux ruisselées sur les versants. 
Tableau 1 Données chimiques et isotopiques pour l'épisode hydrologique incluant la crue du 9 décembre 1990.

\begin{tabular}{|c|c|c|c||c|c|c|c|}
\hline Prélèvements & Débit (1/s) & $\mathrm{Cl}^{-}(\mathrm{mg} / \mathrm{l})$ & ${ }^{18} \mathrm{O}(\%)$ & Prélèvements & Débit (1/s) & $\mathrm{Cl}^{-}(\mathrm{mg} / \mathrm{l})$ & ${ }^{18} \mathrm{O}(\%)$ \\
\hline $29 / 1114 \mathrm{~h} 35$ & 225 & 13,4 & $-7,36$ & $08 \mathrm{~h} 28$ & 567 & 10,0 & $-8,31$ \\
$06 / 1214 \mathrm{~h} 45$ & 32 & 16,0 & $-6,71$ & $08 \mathrm{~h} 58$ & 2857 & 5,8 & $-10,08$ \\
$08 / 1223 \mathrm{~h} 58$ & 36 & 15,5 & $-6,66$ & $09 \mathrm{~h} 28$ & 4395 & 4,1 & $-11,41$ \\
$09 / 1200 \mathrm{~h} 58$ & 58 & 15,0 & $-6,73$ & $09 \mathrm{~h} 58$ & 2011 & 5,9 & $-10,56$ \\
$01 \mathrm{~h} 58$ & 56 & 15,1 & $-6,64$ & $10 \mathrm{~h} 28$ & 1310 & 7,5 & $-9,90$ \\
$02 \mathrm{~h} 58$ & 50 & 15,3 & $-6,78$ & $10 \mathrm{~h} 58$ & 1040 & 8,4 & $-9,70$ \\
$03 \mathrm{~h} 58$ & 47 & 15,6 & $-6,64$ & $11 \mathrm{~h} 28$ & 1154 & 8,4 & $-9,90$ \\
$04 \mathrm{~h} 58$ & 46 & 15,6 & $-6,63$ & $15 \mathrm{~h} 20$ & 672 & 10,1 & $-9,09$ \\
$05 \mathrm{~h} 58$ & 63 & 14,7 & $-7,03$ & $23 \mathrm{~h} 00$ & 376 & & $-8,52$ \\
$06 \mathrm{~h} 58$ & 213 & 12,4 & $-7,47$ & $13 / 1210 \mathrm{~h} 10$ & 69 & 13,7 & $-7,94$ \\
$07 \mathrm{~h} 28$ & 1711 & 6,1 & $-9,07$ & $21 / 1216 \mathrm{~h} 35$ & 31 & 15,1 & $-7,75$ \\
$07 \mathrm{~h} 58$ & 618 & 8,4 & $-8,61$ & $29 / 1215 \mathrm{~h} 30$ & 20 & 15,5 & \\
\hline
\end{tabular}

Le traitement de l'hydrogramme en coordonnées semi-logarithmiques (Fig. 5) permet de fixer à $4391 . \mathrm{s}^{-1}$ le débit marquant la fin de l'intervention du ruissellement sur les versants. La turbidité des eaux ne fournit pas d'argument. Elle a diminué de 29,9 g. $1^{-1}$ pour un débit de $4,4 \mathrm{~m}^{3} \cdot \mathrm{s}^{-1}$, à $0,126 \mathrm{~g} \cdot \mathrm{l}^{-1}$ pour un débit de $5391 . \mathrm{s}^{-1}$. Mais elle est ensuite remontée à 1,4 puis $1,7 \mathrm{~g} . \mathrm{l}^{-1}$ (débits de 418 et $3761 . \mathrm{s}^{-1}$ ), sans doute à cause d'une remobilisation progressive des matériaux assez grossiers (sables et graviers) déposés dans le seuil.

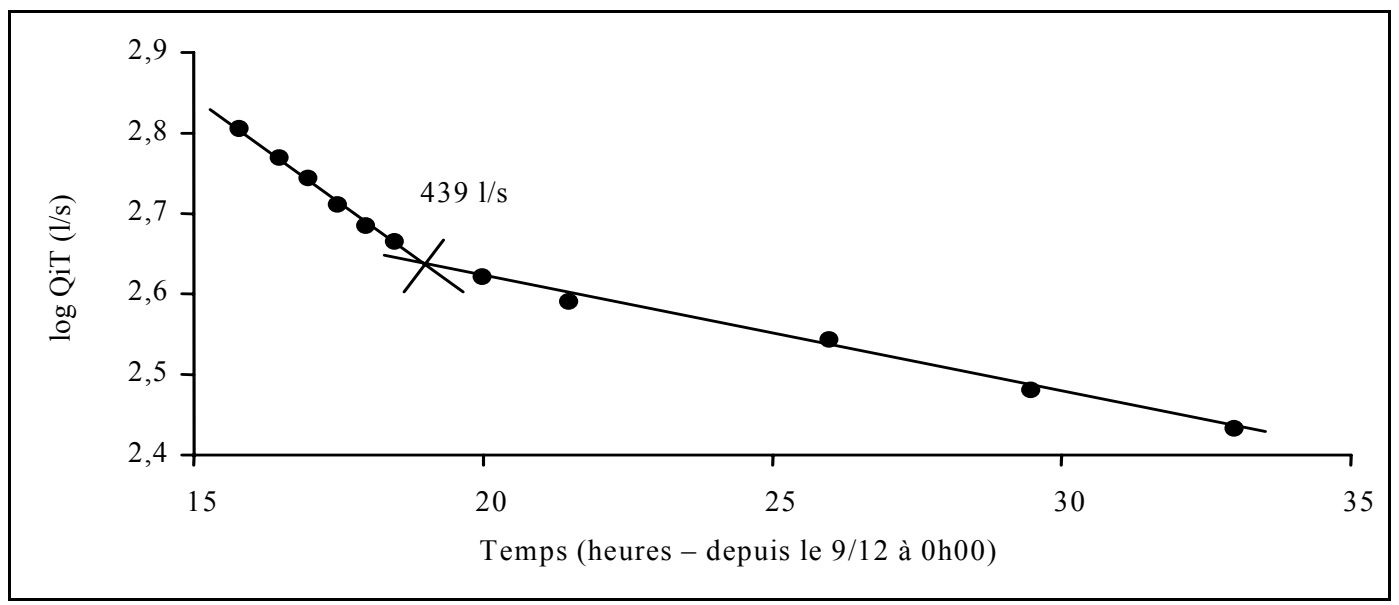

Fig. 5 Débit du ruisseau marquant l'arrêt des écoulements influencés par le ruissellement sur les versants à la suite de la crue du 9 décembre 1990.

Le 8 décembre à 23h47, le ruisseau du Rimbaud avait un débit de 25,9 1.s $\mathrm{s}^{-1}$ avant la pluie. Après la crue, le débit est redescendu à $191 . \mathrm{s}^{-1}$ le 29 décembre à minuit. En extrapolant les courbes de récession jusqu'à un débit de $0,011 . \mathrm{s}^{-1}$, on peut estimer à $6000 \mathrm{~m}^{3}$ environ la différence entre le volume qui, en régime non influencé, se serait écoulé après le 29 décembre et celui qui se serait écoulé à partir du 8 décembre à 23 h47 (on considère que les écarts dus aux différences de pente entre les courbes de tarissement sont certainement négligeables pour les débits inférieurs à $\left.0,011 . \mathrm{s}^{-1}\right)$. Il est en outre possible de corriger les effets des pluies du 11 
au 27 décembre (40,2 mm), en extrapolant les observations satisfaisantes effectuées les 10,11 et 12 décembre (écoulement supplémentaire - par rapport à la courbe de récession en régime non influencé - de près de $15700 \mathrm{~m}^{3}$, provoqué par des précipitations de $14,1 \mathrm{~mm}$ : soit $1095 \mathrm{~m}^{3}$ par mm). L'écoulement généré par l'épisode des 8 et 9 décembre peut être finalement estimé à $112000 \mathrm{~m}^{3}$ environ. Il est cependant possible que cette valeur soit surestimée, car les pluies de la fin du mois ont peut-être atténué la pente de la récession.

La montée de crue débute à la fin de la récession précédente $\left(25,91 . \mathrm{s}^{-1} ; \mathrm{Cl}^{-}\right.$: $16,3 \mathrm{mg} \cdot \mathrm{l}^{-1}$ ). Le débit croît d'abord lentement jusqu'à $631 . \mathrm{s}^{-1}$ (six analyses disponibles, avec des concentrations allant de 15,6 à $\left.14,7 \mathrm{mg} . \mathrm{l}^{-1}\right)$. Les échantillons correspondant à la première montée de crue au-dessus du débit de $631 . \mathrm{s}^{-1}$ (une analyse), à la première décrue (deux analyses, dont une pour un débit de $17111 . \mathrm{s}^{-1}$ ) et à la seconde montée de crue (deux analyses) ne permettent pas de dégager de bonnes régressions entre les concentrations d'ions $\mathrm{Cl}^{-}$et les débits. Les hydrogrammes ont donc été traités par segments délimités par les points de prélèvement et les débits extrêmes. Les six échantillons prélevés au cours de la décrue succédant au second pic de crue (pour des débits compris entre 4395 et $6721 . \mathrm{s}^{-1}$ ) fournissent une très bonne régression bi-logarithmique entre les concentrations d'ions $\mathrm{Cl}^{-}$et les débits $(r$ : $-0,996$ - Fig. 6). L'équation de régression indique des concentrations de 3,09 mg. $\mathrm{l}^{-1}$ en pointe de crue $\left(77901 . \mathrm{s}^{-1}\right)$ et de $12,85 \mathrm{mg} .1^{-1}$ pour le débit de $4391 . \mathrm{s}^{-1}$ marquant la fin de l'intervention du ruissellement sur les versants. Cette dernière valeur a servi (avec les résultats concernant des prélèvements effectués les 13, 21 et 29 décembre) à établir la relation entre le débit $Q i S$ et les concentrations $C S$ durant la deuxième décrue de l'épisode. La valeur de $C S$ au maximum de crue s'établit à 12,33 mg.1 $1^{-1}$ (QiS : $\left.8891 . \mathrm{s}^{-1}\right)$. Associée à la concentration retenue pour le début de l'épisode hydrologique, elle a permis de déterminer l'équation nécessaire au calcul des concentrations $C S$ jusqu'à la première pointe de crue.

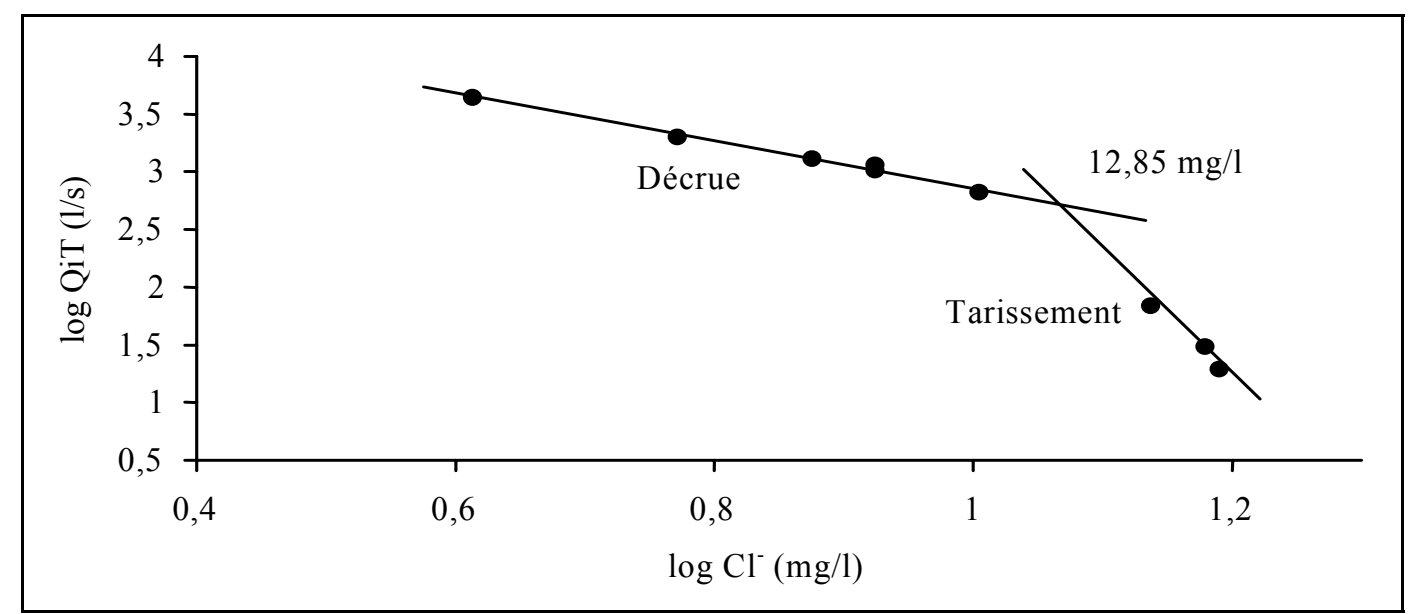

Fig. 6 Relations entre les concentrations en ions chlorures et le débit du ruisseau lors de la récession associée à la crue du 9 décembre 1990.

La décomposition (Fig. 7) fournit de nombreuses informations :

- Écoulement généré par la pluie des 8 et 9 décembre (après retour à $0,011 . \mathrm{s}^{-1}$ ) : $112000 \mathrm{~m}^{3}$ (76,7 mm - coefficient d'écoulement : 87,5\%).

- Écoulement total pendant la période marquée par le ruissellement sur les versants: $50354 \mathrm{~m}^{3}$. 


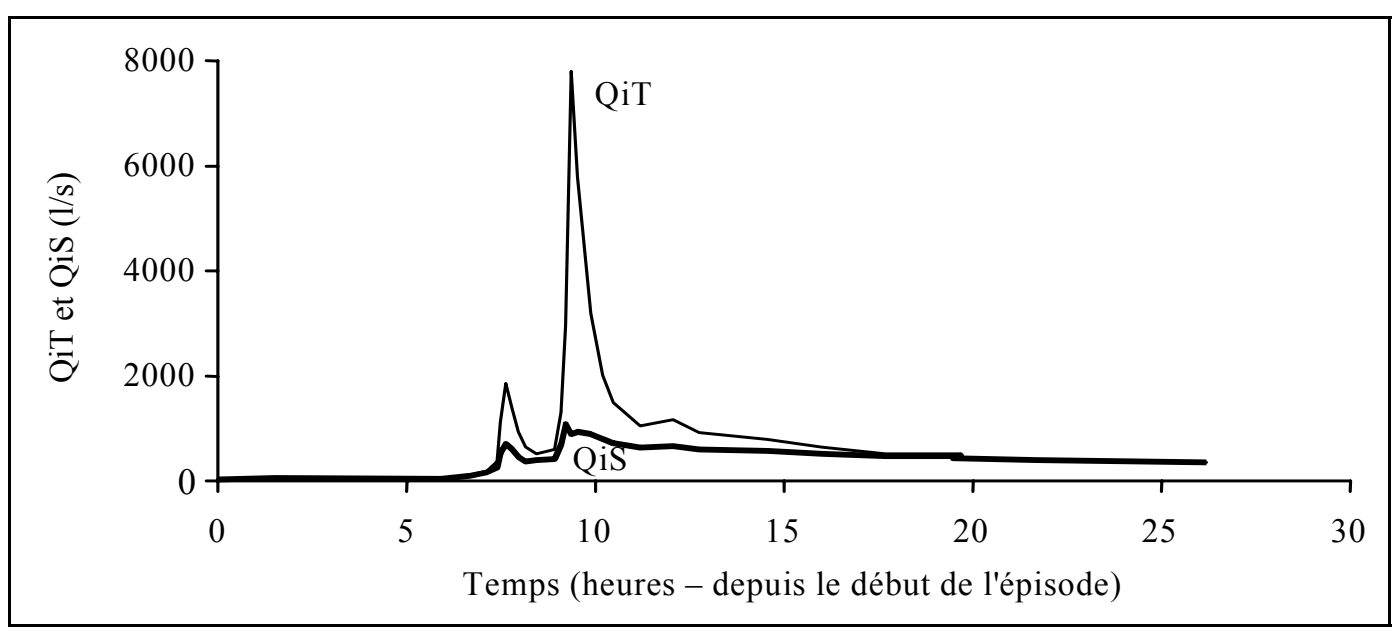

Fig. 7 Décomposition de la crue du 9 décembre 1990.

- Eaux ayant effectué un trajet souterrain : $88372 \mathrm{~m}^{3}(60,5 \mathrm{~mm}-78,9 \%$ de l'écoulement total).

- Eaux fournies par le ruissellement sur les versants : $23634 \mathrm{~m}^{3}(16,2 \mathrm{~mm}-21,1 \%$ de l'écoulement total généré par la pluie des 8 et 9 décembre ; 46,9\% de l'écoulement pendant la seule période concernée par le ruissellement sur les versants).

- Part des eaux fournies par le ruissellement sur les versants au moment de la pointe de crue : $88,6 \%\left(Q i-Q i S=7790-889=69011 . \mathrm{s}^{-1}\right)$. À titre indicatif, notons que dans le cas où la concentration des ions chlorures dans les eaux ayant effectué un trajet souterrain resterait constante pendant la décrue (valeur déterminée pour un débit du ruisseau de $4391 . \mathrm{s}^{-1}$ ), la part du ruissellement sur les versants dans le débit en pointe de crue s'élèverait à $89,1 \%$ (6943 $\left.1 . \mathrm{s}^{-1}\right)$.

- Valeur maximale de QiS : $10911 . \mathrm{s}^{-1}$ (pour un débit QiT de $53701 . \mathrm{s}^{-1}$ en montée de crue ; décomposition peu fiable).

En appliquant aux précipitations du 11-27 décembre le coefficient d'écoulement trouvé pour l'ensemble des écoulements générés par les pluies du 8-27 décembre $(83,6 \%)$, l'écoulement provoqué par la pluie des 8 et 9 décembre serait de 73,3 mm et les eaux de ruissellement sur les versants représenteraient $22,1 \%$ de l'écoulement total au cours de l'épisode.

Un essai de décomposition mené avec les données isotopiques publiées par Travi et al. (1994), en adoptant une approche identique à celle utilisée avec les ions chlorures, donne une valeur de $Q i S$ de $3321 . \mathrm{s}^{-1}$ au maximum de la crue. Ce résultat apparaît d'autant plus douteux que les valeurs trouvées pour les points de prélèvement encadrant le pic de crue sont beaucoup plus élevées : $24921 . \mathrm{s}^{-1}$ pour un QiT de $28571 . \mathrm{s}^{-1}$ en montée de crue (1089 d'après $\mathrm{Cl}^{-}$) et $14261 . \mathrm{s}^{-1}$ pour un QiT de $43951 . \mathrm{s}^{-1}$ en décrue (930 d'après $\left.\mathrm{Cl}^{-}\right)$. Dans l'espoir d'améliorer les résultats, nous avons utilisé, pour la montée de crue, une régression $C S$-QiS établie en remplaçant les données en pointe de crue par celles correspondant au prélèvement effectué pour un débit du ruisseau de $43951 . \mathrm{s}^{-1}$. Les valeurs de QiS s'établissent ainsi à $23711 . \mathrm{s}^{-1}$ pour un $Q i T$ de $2857 \mathrm{1} / \mathrm{s}$, et à $6371 . \mathrm{s}^{-1}$ en pointe de crue. Les faibles écarts entre les concentrations mesurées de l'oxygène-18 (-11,41\%o, le 9 décembre, dans les eaux du 
Rimbaud, pour un débit de $44951 . \mathrm{s}^{-1} ;-7,75 \%$, le 21 décembre, pour un débit de 30,7 1.s ${ }^{-1}$; $-12,47 \%$ dans la pluie) ne permettent pas d'obtenir des résultats satisfaisants.

\section{La crue du 27 septembre 1992}

L'ensemble de cet épisode (Fig. 8) a mis en jeu des précipitations de $72 \mathrm{~mm}$ (dont $69,2 \mathrm{~mm}$ le 27) arrivant sur des sols encore marqués par la sécheresse estivale (précipitations antérieures : 28,0 mm, le 22 septembre). Mais les pluies ont manifesté des intensités très fortes : $140 \mathrm{~mm} \cdot \mathrm{h}^{-1}$ pendant 5 minutes, $120 \mathrm{~mm} \cdot \mathrm{h}^{-1}$ pendant 10 minutes, $68 \mathrm{~mm} \cdot \mathrm{h}^{-1}$ pendant 30 minutes. Le débit en pointe de crue $\left(7,7 \mathrm{~m}^{3} \cdot \mathrm{s}^{-1}\right)$ s'est produit 20 minutes à peine après les précipitations les plus intenses.

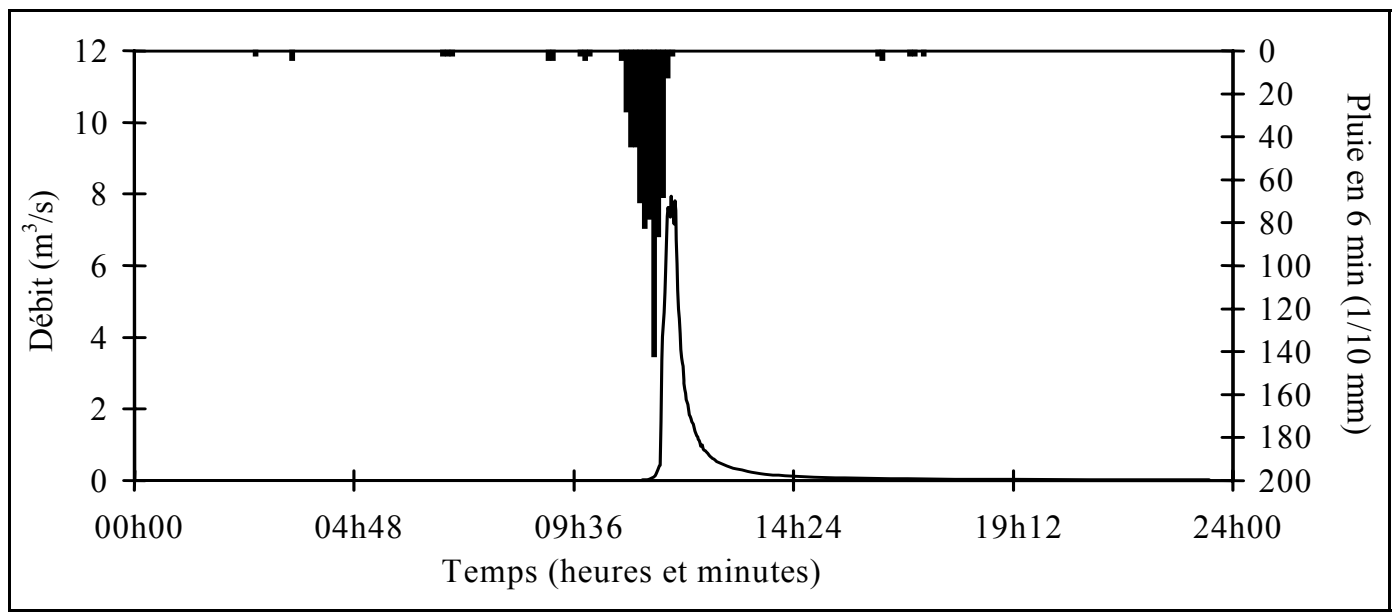

Fig. 8 La crue du 27 septembre 1992.

La détérioration du tuyau en PVC du préleveur automatique d'échantillons ayant enrichi les échantillons d'eau en ions chlorures, la décomposition a été effectuée à partir du sodium (Tableau 2). La comparaison des concentrations de cet élément dans les eaux de pluie (1,29 mg. $\mathrm{l}^{-1}$, au pluviographe de la parcelle) et dans les eaux de ruissellement de la parcelle expérimentale $\left(1,25 \mathrm{mg} .1^{-1}\right.$, sur un terrain où le taux de recouvrement par les arbustes restait inférieur à $5 \%$ - coefficient de ruissellement: $95 \%$ ) autorise cette approche. Le ruissellement sur les versants se développant essentiellement dans les secteurs peu revégétalisés, nous avons affecté à ce type d'écoulement une concentration de 1,27 mg.1 $\mathrm{l}^{-1}$.

Tableau 2 Données chimiques et isotopiques pour l'épisode hydrologique incluant la crue du 27 septembre 1992.

\begin{tabular}{|c|c|c|c|c|c|c|c|}
\hline Prélèvements & Débit (1/s) & $\mathrm{Na}^{+}(\mathrm{mg} / \mathrm{l})$ & ${ }^{18} 0(\%)$ & Prélèvements & Débit (1/s) & $\mathrm{Na}^{+}(\mathrm{mg} / \mathrm{l})$ & ${ }^{18} 0(\%)$ \\
\hline $10 / 0811 \mathrm{~h} 10$ & 0,72 & 9,7 & $-6,63$ & $12 \mathrm{~h} 54$ & 405 & 5,0 & $-5,25$ \\
$27 / 0911 \mathrm{~h} 24$ & 207 & 2,5 & $-4,95$ & $13 \mathrm{~h} 24$ & 229 & 6,1 & $-5,13$ \\
$11 \mathrm{~h} 54$ & 4407 & 2,2 & $-5,67$ & $28 / 0916 \mathrm{~h} 35$ & 5,3 & 10,4 & $-5,28$ \\
$12 \mathrm{~h} 24$ & 945 & 3,6 & $-5,37$ & & & & \\
\hline
\end{tabular}


La représentation de l'hydrogramme en coordonnées semi-logarithmiques (Fig. 9) a permis d'estimer à $1361 . \mathrm{s}^{-1}$ le débit du ruisseau pour lequel les eaux écoulées n'étaient pas influencées par le ruissellement sur les versants. La pluie avait alors cessé depuis un peu plus de deux heures. Pour étayer ce choix, ajoutons que la concentration des suspensions était encore de $97 \mathrm{mg} . \mathrm{l}^{-1}$ une heure trois quarts après la fin des pluies, pour un débit de $2291 . \mathrm{s}^{-1}$ (concentration maximale mesurée au cours de l'épisode : $3,7 \mathrm{~g} \cdot 1^{-1}-$ pour un débit de $4,4 \mathrm{~m}^{3} \cdot \mathrm{s}^{-1}$ en décrue).

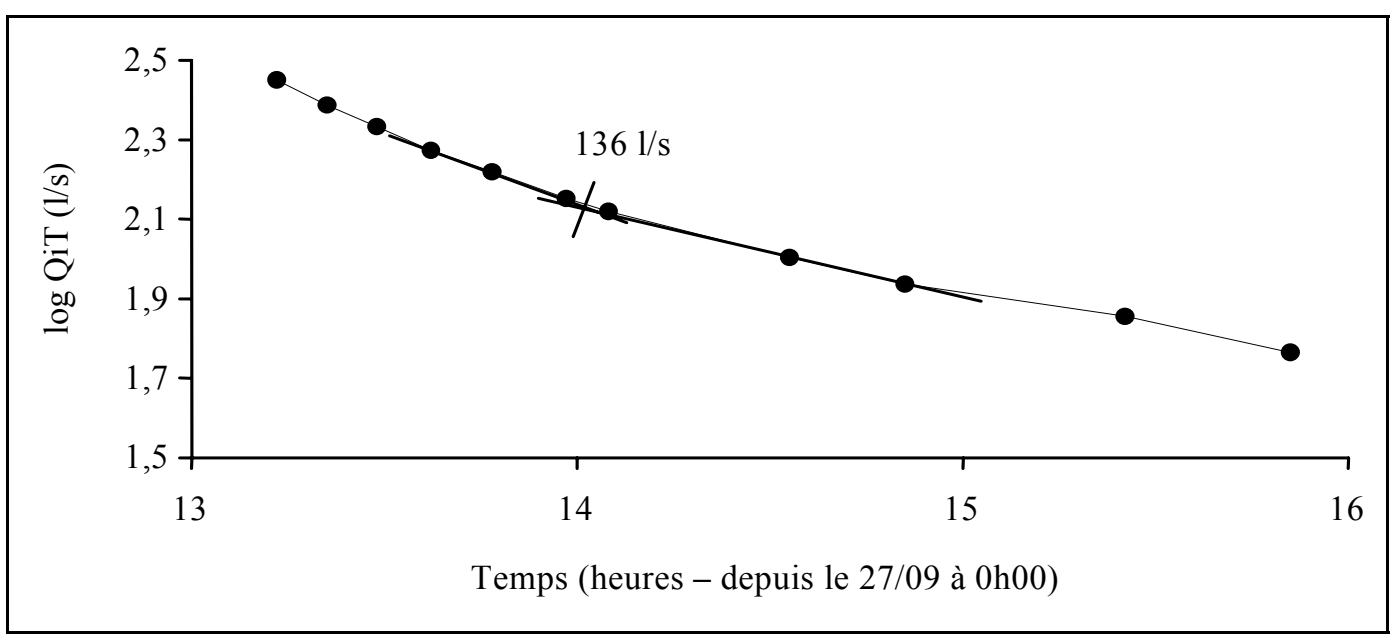

Fig. 9 Débit du ruisseau marquant l'arrêt des écoulements influencés par le ruissellement sur les versants à la suite de la crue du 27 septembre 1992.

Avant la crue, le débit du Rimbaud était de $0,011 . \mathrm{s}^{-1}$ le 26 septembre à minuit. Après la crue, il est retombé à $0,91 . \mathrm{s}^{-1}$ le 2 octobre à minuit. En extrapolant la courbe de récession, on peut estimer à $693 \mathrm{~m}^{3}$ le volume qui se serait écoulé pour que le débit revienne à $0,011 . \mathrm{s}^{-1}$ en régime non influencé.

Nous avons fait correspondre le début de la montée de crue à un débit de $0,721 . \mathrm{s}^{-1}$ (concentration retenue : 9,7 mg. $\mathrm{l}^{-1}$ - mesurée le 10 août 1991 pour le même débit). Les quatre prélèvements effectués pendant la décrue fournissent une régression de très bonne qualité entre les concentrations du sodium et les débits $(r=-0,9991-$ Fig. 10). En pointe de crue, la concentration du sodium aurait avoisiné $1,79 \mathrm{mg} . \mathrm{l}^{-1}$. Pour le débit de $1361 . \mathrm{s}^{-1}$, la concentration du sodium calculée à l'aide de l'équation de régression, s'élève à 7,23 mg. $1^{-1}$. Cette valeur a servi à déterminer la régression concentrations-débits pour la deuxième partie de la récession (prélèvement à 5,3 $\left.1 . \mathrm{s}^{-1} ; \mathrm{Na}^{+}: 10,4 \mathrm{mg} . \mathrm{l}^{-1}\right)$. En pointe de crue $\left(77321 . \mathrm{s}^{-1}\right)$, les eaux ayant effectué un parcours souterrain $(Q i S)$ représentaient $8701 . \mathrm{s}^{-1}$, et leur concentration en sodium était de 5,88 mg. $\left.1^{-1}\right)$. Pour la montée de crue, nous disposons d'un prélèvement pour un débit de $2071 . \mathrm{s}^{-1}\left(\mathrm{Na}^{+}: 2,5 \mathrm{mg} \cdot \mathrm{l}^{-1}\right)$.

La décomposition (Fig. 11) aboutit aux résultats suivants :

- Écoulement généré par la pluie du 27 septembre (après retour à $0,011 . \mathrm{s}^{-1}$ ) : $19030 \mathrm{~m}^{3}$ (13,0 mm - coefficient d'écoulement : 18,6 \%).

- Écoulement total pendant la période marquée par le ruissellement sur les versants: $15713 \mathrm{~m}^{3}$.

- Eaux ayant effectué un trajet souterrain : $7046 \mathrm{~m}^{3}$ (4,8 mm - 37,0\% de l'écoulement total). 


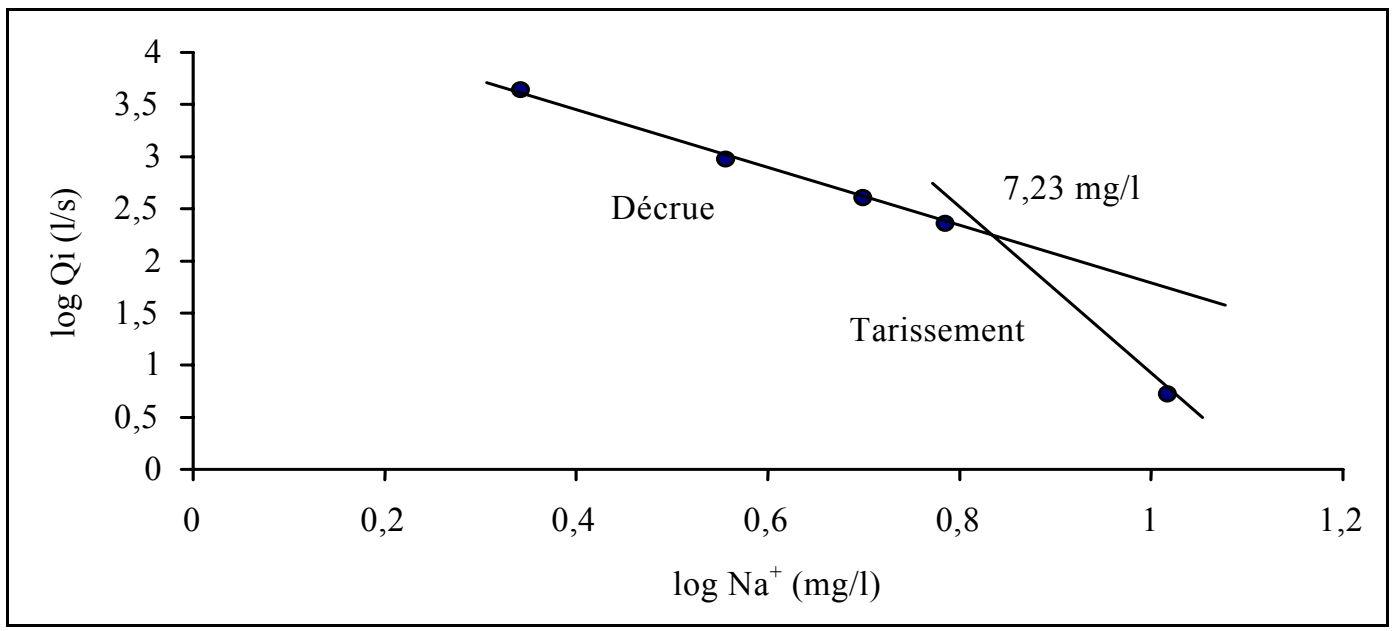

Fig. 10 Relations entre les concentrations en sodium et le débit du ruisseau lors de la récession associée à la crue du 27 septembre 1992.

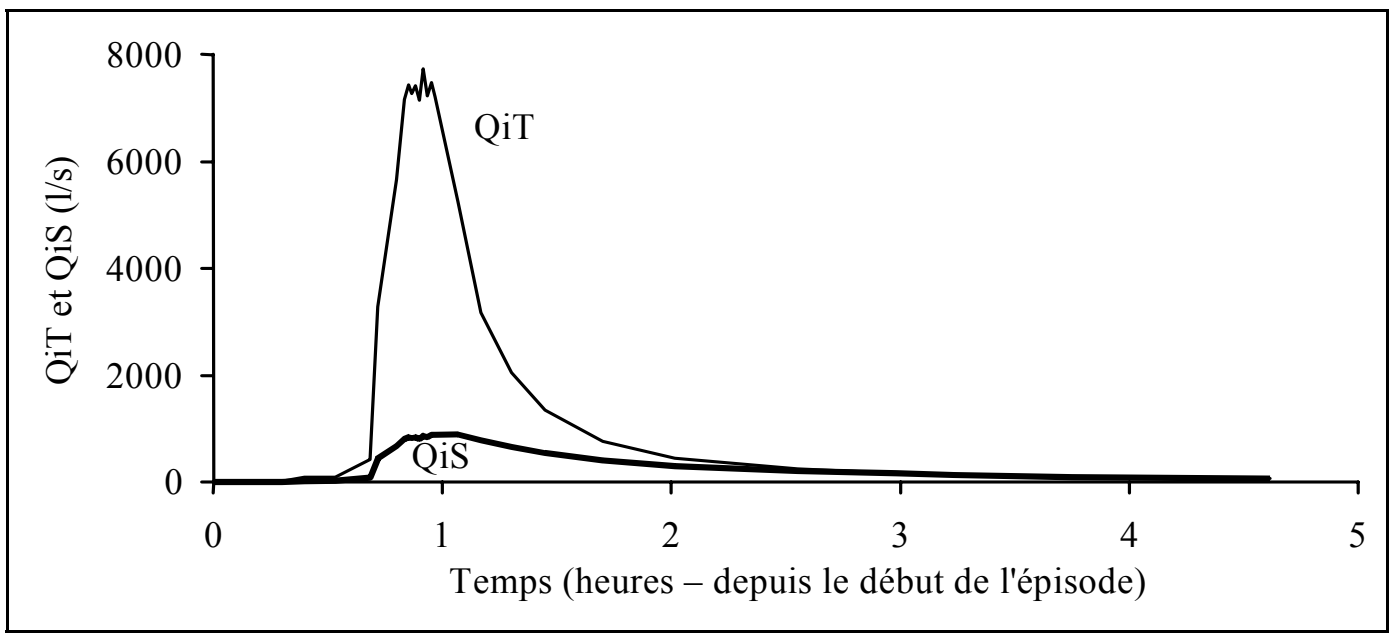

Fig. 11 Décomposition de la crue du 27 septembre 1992.

- Eaux fournies par le ruissellement sur les versants : $11984 \mathrm{~m}^{3}(8,2 \mathrm{~mm}-63,0 \% \mathrm{de}$ l'écoulement total généré par la pluie du 27 septembre ; 76,3\% de l'écoulement pendant la seule période concernée par le ruissellement sur les versants).

- Part des eaux fournies par le ruissellement sur les versants au moment de la pointe de crue : $88,7 \%\left(Q i-Q i S=7732-870=68621 . \mathrm{s}^{-1}\right)$. Dans l'hypothèse où, pendant la décrue, la concentration du sodium resterait constante dans les eaux ayant effectué un trajet souterrain (valeur déterminée pour un débit du ruisseau de $1361 . \mathrm{s}^{-1}$ ), la part du ruissellement sur les versants dans le débit de pointe de crue atteindrait 91,3\% $\left(70571 . \mathrm{s}^{-1}\right)$.

- Valeur maximale de QiS : 895 1/s (pour un débit QiT de 6526 1/s en décrue).

Les analyses isotopiques réalisées pour cet épisode sont inexploitables, car les teneurs en ${ }^{18} \mathrm{O}$ des eaux du Rimbaud ont peu varié au cours de la crue $(-4,95$ à $-5,28 \%$ ) et qu'elles se sont en outre révélées supérieures à la fois au signal "entrée" (-5,86\%o dans la pluie, $-6,05 \%$ dans les eaux recueillies à la parcelle expérimentale) et à la teneur mesurée avant la crue dans les eaux du ruisseau $(-6,63 \%)$. 


\section{CONCLUSION}

En détruisant le couvert végétal, l'incendie a provoqué une augmentation de la fréquence des crues. Le ruisseau du Rimbaud, qui était déjà très réactif aux précipitations avant le feu, a adopté un comportement impulsionnel caractérisé par des réponses hydrologiques très violentes, mais brèves.

Les eaux ruisselant à la surface des versants incendiés ont montré des concentrations en sodium et en ions chlorures identiques à celles des eaux de pluie. Ces éléments ont donc servi à la décomposition des hydrogrammes de crue entre les eaux fournies par le ruissellement sur les versants et les eaux ayant effectué un trajet souterrain. L'approche adoptée tient compte de l'évolution des concentrations dans les différents types d'eau alimentant le ruisseau au cours de l'épisode. Elle repose sur la connaissance de la concentration du marqueur dans les eaux de pluie, et sur l'utilisation de régressions entre les concentrations et les débits pour les différentes parties des hydrogrammes.

Les décompositions confirment l'influence des écoulements directement issus de la surface des versants sur la genèse des crues après l'incendie. Certes, les conditions hydrologiques antérieures à l'épisode déterminent l'abondance de la totalité de l'écoulement généré par les pluies - nettement supérieur en décembre $1990(76,7 \mathrm{~mm}$, pour 87,7 $\mathrm{mm}$ de précipitations), après un automne assez humide, qu'en septembre 1992 (13,0 $\mathrm{mm}$, pour $72,0 \mathrm{~mm}$ de précipitations), en tout début d'année hydrologique - et les eaux d'origine souterraine y tiennent une place plus importante $(78,9 \%$, contre $37,0 \%$ seulement). Mais les crues elles-mêmes sont toujours fortement influencées par le ruissellement sur les versants : les eaux superficielles représentent $46,9 \%$ de l'écoulement pendant la période où le ruisseau a évacué des eaux de ce type lors de la crue de décembre 1990, et 76,3\% lors de celle de septembre 1992. En pointe de crue, la part des eaux superficielles avoisine $89 \%$ dans les deux cas.

La crue du 27 septembre 1992 s'est produite alors que le bassin versant était déjà partiellement revégétalisé. Sa violence $\left(7,7 \mathrm{~m}^{3} \cdot \mathrm{s}^{-1}\right.$; contre $7,8 \mathrm{~m}^{3} \cdot \mathrm{s}^{-1}$ le 9 décembre 1990) est liée à l'intensité des pluies $\left(120 \mathrm{~mm} \cdot \mathrm{h}^{-1}\right.$ pendant 10 minutes - alors qu'elle a atteint seulement $40 \mathrm{~mm} \cdot \mathrm{h}^{-1}$ en 6 minutes en décembre 1990). Des pointes de crue bien plus spectaculaires ont été observées avant l'incendie (Fig. 2)), en particulier en septembre 1968 (plus de $15 \mathrm{~m}^{3} . \mathrm{s}^{-1}$ ) pour des précipitations très violentes (une averse totalisant $110 \mathrm{~mm}$ en $1 \mathrm{~h} 15$ ). En effet, dans certaines parties du bassin, le couvert végétal et les formations superficielles se sont toujours révélés insuffisants pour amortir la réponse hydrologique lorsque les précipitations sont très intenses et suffisamment abondantes. Les secteurs situés à proximité de la partie moyenne des ruisseaux, sur des versants très raides où les gneiss affleurent largement, jouent à cet égard un rôle essentiel. En septembre 1992, leur revégétalisation était trop modeste pour empêcher que se produise un ruissellement aussi fort que celui observé sur la parcelle expérimentale (coefficient d'écoulement mesuré le 27 septembre : $95 \%$ ).

Remerciements : Les recherches ont été effectuées dans le cadre du GIS Réal Collobrier, avec des financements du Conseil Régional de la Région Provence-Alpes-Côte d'Azur et de la CEE (programme européen DM2E). Les dosages du sodium pour la crue du 27 septembre 1992 ont été effectués par J. Quillard (Centre de Géomorphologie du CNRS, Caen), et ceux de l'oxygène-18, pour les deux crues, par M. Daniel (Laboratoire d'Hydrogéologie, Avignon). 


\section{RÉFÉRENCES}

Blavoux, B., Dray, M. \& Merot, Ph. (1976) Composition des écoulements sur deux bassins versants élémentaires, bocager et "ouvert", à l'aide du traçage naturel par ${ }^{18} \mathrm{O}$. Ecosystèmes Bocages, CR CNRS, 153-158.

Hooper, R.P. \& Schoemaker, C.A. (1986) A comparison of isotopic and chemical hydrograph separation. Wat. Resour. Res., 22, 1444-1454.

Lavabre, J., Sempere-Torres, D. \& Cernesson, F. (1993) Hydrological consequences of fire: changes on the hydrological response of a little Mediterranean basin a year after the fire. J. Hydrol., 142, 273-299.

Lavabre, J., Arnaud, P., Folton, N. \& Michel, C. (1997) Les écoulements d'un petit bassin versant méditerranéen après un incendie de forêt. Ingénieries - EAT, 7, 21-30.

Martin, C. (1987) Les mesures de l'érosion chimique dans les bassins versants de roches cristallines: comparaison des résultats par différentes méthodes d'investigation dans le massif des Maures (Var - France). Z. Geomorph., N.F., 31 (1), 73-84.

Martin, C. (1995) Les conséquences hydrochimiques de l'incendie de forêt d'août 1990 dans le massif des Maures. Ann. de Géogr., 581/582, 182-187.

Martin, C., Lavabre, J., Travi, Y., Gimenez, H., Quillard, J., Daniel, M. \& Chevalier, Y. (1994) Comportement impulsionnel du ruisseau du Rimbaud à la suite d'un incendie de forêt : l'exemple de la crue du 27 septembre 1992. Ét. Géogr. Phys., XXIII (Actes de la $3^{\text {ème }}$ Journée Scientifique du GIS Réal Collobrier, Avignon, 1993), 13-14.

Martin, C., Bernard-Allée, Ph., Béguin, É., Levant, M. \& Quillard, J. (1995) Conséquences de l'incendie de forêt de l'été 1990 sur l'érosion mécanique des sols dans le massif des Maures. Bull. Assoc. Géogr. Franç., 5, 438-447.

Michel, C., Edijatno \& Leviandier, T. (1991) Progrès et application de la modélisation conceptuelle pluie-débit. Rapport quadriennal 1987-1990 pour la XXème Assemblée Générale de l'Union Géodésique et Géophysique Internationale (Vienne, 1991), Publié avec le concours de l'Académie des Sciences de Paris, 219-222.

Puech, C., Lavabre, J. \& Martin, C. (1991) Les feux de forêt de l'été 1990 dans le massif des Maures : cartographie à l'aide de l'imagerie satellitaire, premières conséquences sur le cycle hydrologique, recherche sur les phénomènes d'érosion. Sécheresse, 2, 175-181.

Puech, C., Viné, P. \& Leibreich, J. (1994) Évolution comparée entre variations d'écoulement et couvert végétal suite à incendie. Réal Collobrier 1990-1993. Actes du Workshop Final du Projet Européen DM2E (Barcelone, 1994), Édit. Cemagref, Aix-en-Provence.

Travi, Y., Lavabre, J., Blavoux, B. \& Martin, C. (1994) Traçage chimique et isotopique $\left(\mathrm{Cl}^{-}\right.$et ${ }^{18} \mathrm{O}$ ) d'une crue d'automne sur un petit bassin versant méditerranéen incendié. Journal des Sciences Hydrologiques, 39 (6), 605-619.

Viné, P. \& Puech, C. (1994) Suivi, par télédétection, de la régénération forestière après incendie et étude de l'influence de la végétation sur les écoulements. Premiers résultats. Ét. Géogr. Phys., XXIII (Actes de la $3^{\text {ème }}$ Journée Scientifique du GIS Réal Collobrier, Avignon, 1993), 33-44. 